Kato expansion in quantum canonical perturbation theory

\title{
Kato expansion in quantum canonical perturbation theory
}

\author{
Nikolaev A. S. ${ }^{1,2}$ \\ 1) Institute of Computing for Physics and Technology, Protvino, Moscow reg., \\ Russia $a^{\mathrm{a})}$ \\ ${ }^{2)}$ RDTeX LTD, Moscow, Russiab)
}

\begin{abstract}
This work establishes a connection between canonical perturbation series in quantum mechanics and a Kato expansion for the resolvent of the Liouville superoperator. Our approach leads to an explicit expression for a generator of a block-diagonalizing Dyson's ordered exponential in arbitrary perturbation order. Unitary intertwining of perturbed and unperturbed averaging superprojectors allows for a description of ambiguities in the generator and block-diagonalized Hamiltonian.

We compare the efficiency of the corresponding computational algorithm with the efficiencies of the Van Vleck and Magnus methods for high perturbative orders.
\end{abstract}

PACS numbers: 11.15.Bt, 02.30.Mv, 31.15.Md, 03.65.Fd

Keywords: quantum canonical perturbation theory, quantum Liouville superoperator, resolvent, Kato expansion

\footnotetext{
a) http://andreysnikolaev.wordpress.com

b) Electronic mail: Andrey.Nikolaev@rdtex.ru
} 
Kato expansion in quantum canonical perturbation theory

\section{INTRODUCTION.}

Canonical Perturbation Theory (CPT) was historically the first perturbation theory in quantum mechanics ${ }^{1}$. It iteratively constructs a unitary transformation of the Hamiltonian matrix into a block-diagonal form. The resulting effective Hamiltonian ${ }^{2,3}$ then allows for a solution of the eigenvalue problem.

Nowadays, Van Vleck ${ }^{4}$ CPT in Primas' superoperatorial formalism ${ }^{5}$ is widely used in quantum chemistry and molecular spectroscopy ${ }^{6,7}$. Contemporary precise models demand time-consuming computations up to very high perturbative orders ${ }^{8}$. This is why new efficient algorithms are important.

We present here a noniterative construction of the block-diagonalizing transformation for CPT. Our approach follows the recent application of Kato perturbation expansion to classical mechanics ${ }^{9}$. It uses the remarkable analogies between mathematical formalisms of perturbation expansions in classical and quantum mechanics.

The method is based on a Kato series ${ }^{10}$ for the the Laurent coefficients of the resolvent of the Liouville superoperator and the Dyson's ordered exponentials. We demonstrate a regular structure in perturbation series and derive an explicit expression for the generator of a block-diagonalizing transformation in any perturbation order:

$$
\hat{G}=i \hat{\mathcal{S}}_{\hat{H}} \hat{H}_{1}
$$

Here, $\hat{\mathcal{S}}_{H}$ is the partial pseudo-inverse of the perturbed Liouville superoperator. Unitary intertwining of perturbed and unperturbed averaging superprojectors allows for a description of ambiguities in the generator and block-diagonalized Hamiltonian. The corresponding perturbative algorithms are sufficiently efficient even for high-order computations.

Note that all calculations here are only formal, in a sense that neither a discussion of power series convergence nor conditions for the existence of constructed superoperators are present.

The downloadable "Supplementary data files" ${ }^{11}$ contain demonstrations and large formulae. The demonstrations use the freeware computer algebra system FORM ${ }^{12}$. 
Kato expansion in quantum canonical perturbation theory

\section{BASIC PERTURBATION SUPEROPERATORS.}

Consider the Hamiltonian $\hat{H}$ that differs from an exactly solvable one by a perturbation:

$$
\hat{H}=\hat{H}_{0}+\alpha \hat{H}_{1}
$$

We will discuss here only time-independent Hamiltonians that have a discrete spectrum.

The goal of canonical perturbation theory is to transform the perturbed Hamiltonian into a simpler block-diagonal operator by a near-identity unitary transformation. Such transformations may be handled conveniently using superoperator formalism ${ }^{5,13}$. Here we will outline only some basics of it.

For any $\hat{G}$, one may introduce a quantum Liouville superoperator acting on the operator space:

$$
\hat{\mathcal{L}}_{\hat{G}}=[\hat{G}, .], \quad \hat{\mathcal{L}}_{\hat{G}} \hat{F}=[\hat{G}, \hat{F}]
$$

We will use the bold calligraphic-style letters here to denote the superoperators. Due to the Jacobi identity, the Liouvillian is the "derivation" of a commutator:

$$
\hat{\mathcal{L}}_{\hat{G}}[\hat{F}, \hat{H}]=\left[\hat{\mathcal{L}}_{\hat{G}} \hat{F}, \hat{H}\right]+\left[\hat{F}, \hat{\mathcal{L}}_{\hat{G}} \hat{H}\right]
$$

The $\hat{\mathcal{L}}_{\hat{H}_{0}}$ of the discrete spectrum system admits the decomposition of its domain into the direct sum $\mathfrak{N} \oplus \mathfrak{R}$, where $\mathfrak{N}$ or $\mathfrak{R}$ are the kernel and the range space, respectively. In order to realize this decomposition, Primas $^{5}$ introduced the following averaging (block-diagonalizing) superoperator:

$$
\hat{\mathcal{P}}_{\hat{H}_{0}} \hat{F}=\lim _{\lambda \rightarrow+0} \lambda \int_{0}^{+\infty} e^{-\lambda t} e^{\frac{i}{\hbar} t \hat{H}_{0}} \hat{F} e^{-\frac{i}{\hbar} t \hat{H}_{0}} d t=\lim _{\lambda \rightarrow+0} \lambda \int_{0}^{+\infty} d t e^{-\lambda t} e^{\frac{i}{\hbar} t \hat{\mathcal{L}}_{\hat{H}_{0}} \hat{F}} .
$$

This superoperator is the projector $\hat{\mathcal{P}}_{\hat{H}_{0}}^{2}=\hat{\mathcal{P}}_{\hat{H}_{0}}$. It projects the operator $\hat{F}$ onto the kernel space $\mathfrak{N}$ of block-diagonal operators commuting with $\hat{H}_{0}$.

The complementary projector $1-\hat{\mathcal{P}}_{\hat{H}_{0}}$ extracts the non-commuting with the $\hat{H}_{0}$ (offdiagonal) part of the $\hat{F}$. It projects on the range space $\mathfrak{R}$ where the inverse of $\hat{\mathcal{L}}_{\hat{H}_{0}}$ exists. This inverse is the integrating superoperator $\hat{\mathcal{S}}_{H_{0}}$, which is also called the solution of the homological equation, tilde operation, zero-mean antiderivative, Friedrichs $\widehat{\Gamma}$ operation, $\frac{\mathbf{1}}{\mathbf{k}}$ operator, division operation, etc. Its invariant definition by Primas ${ }^{5}$ is as follows:

$$
\hat{\mathcal{S}}_{\hat{H}_{0}}=-\frac{i}{\hbar} \lim _{\lambda \rightarrow+0} \int_{0}^{\infty} d t e^{-\lambda t} e^{\frac{i}{\hbar} t \hat{\mathcal{L}}_{\hat{H}_{0}}}\left(1-\hat{\mathcal{P}}_{\hat{H}_{0}}\right) .
$$


Kato expansion in quantum canonical perturbation theory

It is easy to check that:

$$
\begin{aligned}
& \hat{\mathcal{L}}_{\hat{H}_{0}} \hat{\mathcal{S}}_{\hat{H}_{0}}=\hat{\mathcal{S}}_{\hat{H}_{0}} \hat{\mathcal{L}}_{\hat{H}_{0}}=1-\hat{\mathcal{P}}_{\hat{H}_{0}}, \\
& \hat{\mathcal{S}}_{\hat{H}_{0}} \hat{\mathcal{P}}_{\hat{H}_{0}}=\hat{\mathcal{P}}_{\hat{H}_{0}} \hat{\mathcal{S}}_{\hat{H}_{0}} \equiv 0
\end{aligned}
$$

The superoperators $\left(\hat{\mathcal{L}}_{\hat{H}_{1}}, \hat{\mathcal{P}}_{\hat{H}_{0}}, \hat{\mathcal{S}}_{\hat{H}_{0}}\right)$ are the building blocks of the canonical perturbation series $^{5}$. Quantum mechanics uses the following methods for their computation:

- The Energy Representation of an unperturbed Hamiltonian provides the eigenbasis of the Liouville superoperator ${ }^{13}$. The action of the basic superoperators on any operator $\hat{F}$ can be directly computed in this representation as follows:

$$
\hat{\mathcal{P}}_{\hat{H}_{0}} \hat{F}=\sum_{E_{m}=E_{n}} F_{m n}|m\rangle\left\langle n\left|, \quad \hat{\mathcal{S}}_{\hat{H}_{0}} \hat{F}=\sum_{E_{m} \neq E_{n}} \frac{F_{m n}}{E_{m}-E_{n}}\right| m\right\rangle\langle n| .
$$

These well-known expressions are used frequently as the definitions of $\hat{\mathcal{P}}_{\hat{H}_{0}}$ and $\hat{\mathcal{S}}_{\hat{H}_{0}}$.

- The method of Contact Transformations ${ }^{14}$ (quantum Birkhoff-Gustavson normal form ${ }^{15,16}$ ) applies to the perturbed harmonic oscillator

$$
\hat{H}=\sum_{k=1}^{\mathbf{d}} \frac{\omega_{k}}{2}\left(\hat{p}_{k}^{2}+\hat{q}_{k}^{2}\right)+\alpha \hat{H}_{1}(\hat{p}, \hat{q}) .
$$

It uses the representation of ladder operators:

$$
\hat{q}_{k}=\sqrt{\frac{\hbar}{2}}\left(\hat{a}_{k}^{\dagger}+\hat{a}_{k}\right), \quad p_{k}=i \sqrt{\frac{\hbar}{2}}\left(\hat{a}_{k}^{\dagger}-\hat{a}_{k}\right), \quad\left[\hat{a}_{k}, \hat{a}_{l}^{\dagger}\right]=\delta_{k l} .
$$

The action of basic superoperators on a Wick-ordered polynomial operator $\hat{F}(\hat{p}, \hat{q})=$ $\sum \tilde{F}(\vec{m}, \vec{n}) \hat{a}^{\dagger \vec{m}} \hat{a}^{\vec{n}}$ is as follows:

$$
\hat{\mathcal{P}}_{\hat{H}_{0}} \hat{F}=\sum_{(\vec{\omega}, \vec{m}-\vec{n})=0} \tilde{F}(\vec{m}, \vec{n}) \hat{a}^{\dagger \vec{m}} \hat{a}^{\vec{n}}, \quad \hat{\mathcal{S}}_{\hat{H}_{0}} \hat{F}=\sum_{(\vec{\omega}, \vec{m}-\vec{n}) \neq 0} \frac{\tilde{F}(\vec{m}, \vec{n})}{\hbar(\vec{\omega}, \vec{m}-\vec{n})} \hat{a}^{\dagger \vec{m}} \hat{a}^{\vec{n}} .
$$

\section{THE SUPERRESOLVENT}

Consider the resolvent of the Liouville superoperator:

$$
\hat{\mathscr{R}}_{H}(z)=\frac{1}{\frac{i}{\hbar} \hat{\mathcal{L}}_{H}-z} .
$$

This superoperator-valued function of the complex variable $z$ is the Laplace transform of the evolution superoperator 
Kato expansion in quantum canonical perturbation theory

$$
\hat{\mathscr{R}}_{H}(z)=-\int_{0}^{+\infty} d t e^{-z t} e^{\frac{i}{\hbar} t \hat{\mathcal{L}}_{H}} .
$$

Resolvent singularities are the eigenvalues of the Liouvillian. Typically, its spectrum $\boldsymbol{\sigma}\left(\hat{\mathcal{L}}_{\hat{H}}\right)=\boldsymbol{\sigma}(\hat{H})-\boldsymbol{\sigma}(\hat{H})$ has a richer structure than that of the corresponding Hamiltonian ${ }^{17}$.

Let us begin with the simple case of the pure point spectrum of a quantum system with isolated energy levels. The singularities of corresponding Liouvillian superresolvent are located at $E_{k}-E_{m}$ and are separable from the origin.

The existence of $\hat{\mathcal{P}}_{\hat{H}_{0}}$ and $\hat{\mathcal{S}}_{\hat{H}_{0}}$ means that the unperturbed superresolvent has a simple pole in 0 . The averaging superoperator is its residue in this pole:

$$
\hat{\mathcal{P}}_{\hat{H}_{0}} \equiv-\operatorname{Res}_{z=0} \hat{\mathscr{R}}_{\hat{H}_{0}}
$$

while the integrating superoperator $\hat{\mathcal{S}}_{\hat{H}_{0}}$ is its holomorphic part:

$$
\hat{\mathcal{S}}_{\hat{H}_{0}}=\frac{i}{\hbar} \lim _{z \rightarrow 0} \hat{\mathscr{R}}_{\hat{H}_{0}}(z)\left(1-\hat{\mathcal{P}}_{H_{0}}\right)
$$

Therefore, the Liouvillian superresolvent combines both basic perturbation superoperators ${ }^{9}$. This allows us to apply the powerful formalism of complex analysis to perturbation theory.

It is well known ${ }^{10}$ that, due to the Hilbert resolvent identity

$$
\hat{\mathscr{R}}_{\hat{H}}\left(z_{1}\right)-\hat{\mathscr{R}}_{\hat{H}}\left(z_{2}\right)=\left(z_{1}-z_{2}\right) \hat{\mathscr{R}}_{\hat{H}}\left(z_{1}\right) \hat{\mathscr{R}}_{\hat{H}}\left(z_{2}\right)
$$

the Laurent series for the unperturbed resolvent has the form of a geometric progression

$$
\hat{\mathscr{R}}_{\hat{H}_{0}}(z)=-\frac{1}{z} \hat{\mathcal{P}}_{\hat{H}_{0}}+\sum_{n=0}^{\infty} z^{n}\left(\frac{\hbar}{i} \hat{\mathcal{S}}_{\hat{H}_{0}}\right)^{n+1}=\sum_{n=0}^{+\infty}\left(\frac{\hbar}{i}\right)^{n} \hat{\mathcal{R}}_{H_{0}}^{(n)} z^{n-1} .
$$

Here, we have denoted $\hat{\mathcal{R}}_{H_{0}}^{(0)}=-\hat{\mathcal{P}}_{\hat{H}_{0}}$ and $\hat{\mathcal{R}}_{H_{0}}^{(n)}=\hat{\mathcal{S}}_{\hat{H}_{0}}^{n}$.

The perturbed resolvent may be more singular. The Laurent series for a general resolvent with an isolated singularity at the origin has the following form ${ }^{10}$ :

$$
\hat{\mathscr{R}}_{\hat{H}}(z)=-\frac{1}{z} \hat{\mathcal{P}}_{\hat{H}}+\sum_{n=0}^{\infty} z^{n}\left(\frac{\hbar}{i} \hat{\mathcal{S}}_{\hat{H}}\right)^{n+1}-\sum_{n=2}^{\infty} z^{-n}\left(\frac{i}{\hbar} \hat{\mathcal{D}}_{\hat{H}}\right)^{n-1}
$$

Here, $\hat{\mathcal{D}}_{\hat{H}}$ is the eigen-nilpotent superoperator, which does not have an unperturbed analogue. 
Kato expansion in quantum canonical perturbation theory

\section{A. Kato series}

If the perturbation $\hat{H}_{1}$ is relatively bounded with respect to $\hat{H}_{0}$, then the RayleighSchrödinger series converges ${ }^{10}$ and the perturbed superresolvent can be expanded into the Neumann series as follows:

$$
\begin{aligned}
\hat{\mathscr{R}}_{\hat{H}_{0}+\alpha \hat{H}_{1}}(z) & =\hat{\mathscr{R}}_{\hat{H}_{0}}-\alpha \frac{i}{\hbar} \hat{\mathscr{R}}_{\hat{H}_{0}} \hat{\mathcal{L}}_{\hat{H}_{1}} \hat{\mathscr{R}}_{\hat{H}_{0}}+\alpha^{2}\left(\frac{i}{\hbar}\right)^{2} \hat{\mathscr{R}}_{\hat{H}_{0}} \hat{\mathcal{L}}_{\hat{H}_{1}} \hat{\mathscr{R}}_{\hat{H}_{0}} \hat{\mathcal{L}}_{\hat{H}_{1}} \hat{\mathscr{R}}_{\hat{H}_{0}}+\ldots \\
& =\sum_{n=0}^{\infty}\left(-\alpha \frac{i}{\hbar}\right)^{n} \hat{\mathscr{R}}_{\hat{H}_{0}}(z)\left(\hat{\mathcal{L}}_{\hat{H}_{1}} \hat{\mathscr{R}}_{\hat{H}_{0}}(z)\right)^{n} .
\end{aligned}
$$

The integration around a small contour results in the Kato series ${ }^{10}$ for the "perturbed averaging superoperator":

$$
\begin{aligned}
\hat{\mathcal{P}}_{\hat{H}} & =-\frac{1}{2 \pi i} \oint_{|z|=\epsilon} \hat{\mathscr{R}}_{\hat{H}(z)} d z \\
& =-\frac{1}{2 \pi i} \sum_{n=0}^{\infty} \oint_{|z|=\epsilon}\left(-\alpha \frac{i}{\hbar}\right)^{n} \hat{\mathscr{R}}_{\hat{H}_{0}}(z)\left(\hat{\mathcal{L}}_{\hat{H}_{1}} \hat{\mathscr{R}}_{\hat{H}_{0}}(z)\right)^{n} d z \\
& =\frac{-1}{2 \pi i} \sum_{n=0}^{\infty}\left(-\alpha \frac{i}{\hbar}\right)^{n} \oint_{|z|=\epsilon}\left(\sum_{m=0}^{\infty}\left(\frac{\hbar}{i}\right)^{m} \hat{\mathcal{R}}_{\hat{H}_{0}}^{(m)} z^{m-1}\right)\left(\hat{\mathcal{L}}_{\hat{H}_{1}} \sum_{k=0}^{\infty}\left(\frac{\hbar}{i}\right)^{k} \hat{\mathcal{R}}_{\hat{H}_{0}}^{(k)} z^{k-1}\right)^{n} d z
\end{aligned}
$$

the "perturbed integrating superoperator", and the "perturbed eigen-nilpotent":

$$
\hat{\mathcal{S}}_{\hat{H}}=\frac{1}{2 \pi \hbar} \oint_{|z|=\epsilon} z^{-1} \hat{\mathscr{R}}_{\hat{H}(z)} d z, \quad \hat{\mathcal{D}}_{\hat{H}}=\frac{\hbar}{2 \pi} \oint_{|z|=\epsilon} z \hat{\mathscr{R}}_{\hat{H}(z)} d z .
$$

Only coefficients of $z^{-1}$ in these expansions will contribute to the result:

$$
\begin{aligned}
& \hat{\mathcal{P}}_{\hat{H}}=\sum_{n=0}^{\infty}(-1)^{n+1} \alpha^{n}(\sum_{\substack{\sum_{p_{j}=\mathbf{n}} \\
p_{j} \geq 0}} \hat{\mathcal{R}}_{\hat{H}_{0}}^{\left(p_{n+1}\right)} \underbrace{\hat{\mathcal{L}}_{\hat{H}_{1}} \hat{\boldsymbol{\mathcal { R }}}_{\hat{H}_{0}}^{\left(p_{n}\right)} \ldots \hat{\boldsymbol{\mathcal { R }}}_{\hat{H}_{0}}^{\left(p_{2}\right)} \hat{\mathcal{L}}_{\hat{H}_{1}}}_{n \text { times }} \hat{\boldsymbol{\mathcal { R }}}_{\hat{H}_{0}}^{\left(p_{1}\right)}), \\
& \hat{\mathcal{S}}_{\hat{H}}=\sum_{n=0}^{\infty}(-1)^{n} \quad \alpha^{n}(\sum_{\substack{\sum p_{j}=\mathbf{n}+\mathbf{1} \\
p_{j} \geq 0}} \hat{\boldsymbol{\mathcal { R }}}_{\hat{H}_{0}}^{\left(p_{n+1}\right)} \underbrace{\hat{\mathcal{L}}_{\hat{H}_{1}} \hat{\boldsymbol{\mathcal { R }}}_{\hat{H}_{0}}^{\left(p_{n}\right)} \ldots \hat{\mathcal{R}}_{\hat{H}_{0}}^{\left(p_{2}\right)} \hat{\mathcal{L}}_{\hat{H}_{1}}}_{n \text { times }} \hat{\boldsymbol{\mathcal { R }}}_{\hat{H}_{0}}^{\left(p_{1}\right)}), \\
& \hat{\mathcal{D}}_{\hat{H}}=\sum_{n=1}^{\infty}(-1)^{n+1} \alpha^{n}(\sum_{\substack{\sum p_{j}=\mathbf{n}-\mathbf{1} \\
p_{j} \geq 0}} \hat{\boldsymbol{\mathcal { R }}}_{\hat{H}_{0}}^{\left(p_{n+1}\right)} \underbrace{\hat{\mathcal{L}}_{\hat{H}_{1}} \hat{\boldsymbol{\mathcal { R }}}_{\hat{H}_{0}}^{\left(p_{n}\right)} \ldots \hat{\boldsymbol{\mathcal { R }}}_{\hat{H}_{0}}^{\left(p_{2}\right)} \hat{\mathcal{L}}_{\hat{H}_{1}}}_{n \text { times }} \hat{\boldsymbol{\mathcal { R }}}_{\hat{H}_{0}}^{\left(p_{1}\right)}) \text {. }
\end{aligned}
$$

Because the superoperator $\hat{\mathcal{R}}_{\hat{H}_{0}}^{\left(p_{j}\right)}$ consists of $p_{j}$ superoperators $\hat{\mathcal{S}}_{\hat{H}_{0}}$, the summation in the above expressions should be done by all possible placements of $n$ (or $n+1$, or $n-1)$ 
Kato expansion in quantum canonical perturbation theory

$\hat{\mathcal{S}}_{\hat{H}_{0}}$ in $n+1$ sets. There are $C_{2 n}^{n}$ terms of order $\alpha^{n}$ in $\hat{\mathcal{P}}_{\hat{H}}$ and $C_{2 n+1}^{n}$ terms in $\hat{\mathcal{S}}_{\hat{H}}$. For the Hermitian $\hat{G}$, the operators $\hat{\mathcal{S}}_{\hat{H}} \hat{G}$ and $\hat{\mathcal{D}}_{\hat{H}} \hat{G}$ are anti-Hermitian.

The properties of unperturbed superoperators can be extended to their analytic continuations as follows:

$$
\hat{\mathcal{P}}_{\hat{H}} \hat{H}=\hat{H}, \quad \hat{\mathcal{S}}_{\hat{H}} \hat{\mathcal{L}}_{\hat{H}}=1-\hat{\mathcal{P}}_{\hat{H}}, \quad \hat{\mathcal{L}}_{\hat{H}} \hat{\mathcal{P}}_{\hat{H}}=\hat{\mathcal{P}}_{\hat{H}} \hat{\mathcal{L}}_{\hat{H}}=\hat{\mathcal{D}}_{\hat{H}}, \text { etc. }
$$

For the details, see our work ${ }^{9}$ on an application of the Kato series to classical mechanics.

To avoid misunderstanding, it should be noted that $\hat{\mathcal{P}}_{\hat{H}} \hat{F}$ will not be commuting with the perturbed Hamiltonian. This is because $\hat{\mathcal{L}}_{\hat{H}} \hat{\mathcal{P}}_{\hat{H}}=\hat{\mathcal{D}}_{\hat{H}} \neq 0$ in general. Actually, the "perturbed superprojector" $\hat{\mathcal{P}}_{\hat{H}}$ projects onto some analytic continuation of the algebra of integrals of the unperturbed Hamiltonian. This may not coincide, in general, with the algebra of integrals of the perturbed system, because of a destruction of symmetries. In other words, the zero eigenvalue of the Liouville superoperator may be split by the perturbation.

\section{B. Canonical properties of the Liouvillian superresolvent}

Because $\hat{\mathcal{L}}$ is a derivative, there exists an integration by parts formula for its superresolvent ${ }^{9}$. For any $z_{1}, z_{2}$, and $z_{3}$ outside of the spectrum of $\hat{\mathcal{L}}_{\hat{H}}$ and any operator $\hat{F}$, the following holds true:

$$
\begin{aligned}
\hat{\mathscr{R}}_{\hat{H}}\left(z_{1}\right) \hat{\mathcal{L}}_{\mathscr{\mathscr { R }}_{\hat{H}}\left(z_{2}\right) \hat{F}}-\hat{\mathcal{L}}_{\hat{\mathscr{R}}_{\hat{H}}\left(z_{2}\right) \hat{F}} \hat{\mathscr{R}}_{\hat{H}}\left(z_{3}\right)+\hat{\mathscr{R}}_{\hat{H}}\left(z_{1}\right) \hat{\mathcal{L}}_{\hat{F}} \hat{\mathscr{R}}_{\hat{H}}\left(z_{3}\right) \\
=\left(z_{1}-z_{2}-z_{3}\right) \hat{\mathscr{R}}_{\hat{H}}\left(z_{1}\right) \hat{\mathcal{L}}_{\hat{\mathscr{R}}_{\hat{H}}\left(z_{2}\right) \hat{F}} \hat{\mathscr{R}}_{\hat{H}}\left(z_{3}\right) .
\end{aligned}
$$

This results from the application of the identical superoperator $\hat{\mathscr{R}}_{\hat{H}}\left(z_{1}\right)\left(\frac{i}{\hbar} \hat{\mathcal{L}}_{\hat{H}}-z_{1}\right) \equiv 1$ to the commutator $\hat{\mathcal{L}}_{\hat{\mathscr{R}}_{\hat{H}}\left(z_{2}\right) \hat{F}} \hat{\mathscr{R}}_{\hat{H}}\left(z_{3}\right)$ and the expansion of the Jacobi identity.

Consider the derivative of the Liouvillian superresolvent with respect to the perturbation

$$
\frac{\partial}{\partial \alpha} \hat{\mathscr{R}}_{\hat{H}}(z)=-\frac{i}{\hbar} \hat{\mathscr{R}}_{\hat{H}}(z) \hat{\mathcal{L}}_{\hat{H}_{1}} \hat{\mathscr{R}}_{\hat{H}}(z)
$$

Substituting $z_{1}=z_{3}=z$, and $\hat{F}=\hat{H}_{1}$ into the canonical superresolvent identity (5) yields

$$
\hat{\mathscr{R}}_{\hat{H}}(z) \hat{\mathcal{L}}_{\hat{H}_{1}} \hat{\mathscr{R}}_{\hat{H}}(z)=\hat{\mathcal{L}}_{\hat{\mathscr{R}}_{\hat{H}}\left(z_{2}\right) \hat{H}_{1}} \hat{\mathscr{R}}_{\hat{H}}(z)-\hat{\mathscr{R}}_{\hat{H}}(z) \hat{\mathcal{L}}_{\hat{\mathscr{R}}_{\hat{H}}\left(z_{2}\right) \hat{H}_{1}}-z_{2} \hat{\mathscr{R}}_{\hat{H}}(z) \hat{\mathcal{L}}_{\hat{\mathscr{R}}_{\hat{H}}\left(z_{2}\right) \hat{H}_{1}} \hat{\mathscr{R}}_{\hat{H}}(z) .
$$

Look at the coefficient of $z_{2}^{0}$ in the Laurent series of the above expression:

$$
\frac{\partial}{\partial \alpha} \hat{\mathscr{R}}_{\hat{H}}(z)=\hat{\mathscr{R}}_{\hat{H}}(z) \hat{\mathcal{L}}_{\hat{\mathcal{S}}_{\hat{H}} \hat{H}_{1}}-\hat{\mathcal{L}}_{\hat{\mathcal{S}}_{\hat{H}} \hat{H}_{1}} \hat{\mathscr{R}}_{\hat{H}}(z)-\frac{i}{\hbar} \hat{\mathscr{R}}_{\hat{H}}(z) \hat{\mathcal{L}}_{\hat{\mathcal{P}}_{\hat{H}} \hat{H}_{1}} \hat{\mathscr{R}}_{\hat{H}}(z)
$$


Kato expansion in quantum canonical perturbation theory

Proceeding similarly for coefficients of $z_{2}^{-n}(n \geq 1)$ in $(6)$, we obtain

$$
\begin{aligned}
& \hat{\mathscr{R}}_{\hat{H}}(z) \hat{\mathcal{L}}_{\hat{\mathcal{P}}_{\hat{H}} \hat{H}_{1}}=\hat{\mathcal{L}}_{\hat{\mathcal{P}}_{\hat{H}} \hat{H}_{1}} \hat{\mathscr{R}}_{\hat{H}}(z)-\frac{i}{\hbar} \hat{\mathscr{R}}_{\hat{H}}(z) \hat{\mathcal{L}}_{\hat{\mathcal{D}}_{\hat{H}} \hat{H}_{1}} \hat{\mathscr{R}}_{\hat{H}}(z), \\
& \hat{\mathscr{R}}_{\hat{H}}(z) \hat{\mathcal{L}}_{\hat{\mathcal{D}}_{\hat{H}}^{n} \hat{H}_{1}}=\hat{\mathcal{L}}_{\hat{\mathcal{D}}_{\hat{H}}^{n} \hat{H}_{1}} \hat{\mathscr{R}}_{\hat{H}}(z)-\frac{i}{\hbar} \hat{\mathscr{R}}_{\hat{H}}(z) \hat{\mathcal{L}}_{\hat{\mathcal{D}}_{\hat{H}}^{n+1} \hat{H}_{1}} \hat{\mathscr{R}}_{\hat{H}}(z) .
\end{aligned}
$$

This allows us to rewrite the expression for the superresolvent derivative as

$$
\begin{aligned}
\frac{\partial}{\partial \alpha} \hat{\mathscr{R}}_{\hat{H}}(z)= & \hat{\mathscr{R}}_{\hat{H}}(z) \hat{\mathcal{L}}_{\hat{\mathcal{S}}_{\hat{H}} \hat{H}_{1}}-\hat{\mathcal{L}}_{\hat{\mathcal{S}}_{\hat{H}} \hat{H}_{1}} \hat{\mathscr{R}}_{\hat{H}}(z) \\
& -\frac{i}{\hbar} \hat{\mathcal{L}}_{\hat{\mathcal{P}}_{\hat{H}} \hat{H}_{1}} \hat{\mathscr{R}}_{\hat{H}}(z)^{2}+\left(\frac{i}{\hbar}\right)^{2} \hat{\mathcal{L}}_{\hat{\mathcal{D}}_{\hat{H}} \hat{H}_{1}} \hat{\mathscr{R}}_{\hat{H}}(z)^{3}-\left(\frac{i}{\hbar}\right)^{3} \hat{\mathcal{L}}_{\hat{\mathcal{D}}_{\hat{H}}^{2} \hat{H}_{1}} \hat{\mathscr{R}}_{\hat{H}}(z)^{4}+\ldots
\end{aligned}
$$

Actually, this is a power series because $\hat{\mathcal{D}}_{\hat{H}}^{n}=\mathrm{O}\left(\alpha^{n}\right)$.

It follows from the Hilbert resolvent identity that

$$
\frac{\partial^{n}}{\partial z^{n}} \hat{\mathscr{R}}_{\hat{H}}(z)=n ! \hat{\mathscr{R}}_{\hat{H}}^{n+1}(z)
$$

Finally, we obtain

$$
\begin{aligned}
\frac{\partial}{\partial \alpha} \hat{\mathscr{R}}_{\hat{H}}(z)= & \hat{\mathscr{R}}_{\hat{H}} \hat{\mathcal{L}}_{\hat{\mathcal{S}}_{\hat{H}} \hat{H}_{1}}-\hat{\mathcal{L}}_{\hat{\mathcal{S}}_{\hat{H}} \hat{H}_{1}} \hat{\mathscr{R}}_{\hat{H}}(z)-\frac{i}{\hbar} \hat{\mathcal{L}}_{\hat{\mathcal{P}}_{\hat{H}} \hat{H}_{1}} \frac{\partial \hat{\mathscr{R}}_{\hat{H}}(z)}{\partial z} \\
& +\frac{1}{2}\left(\frac{i}{\hbar}\right)^{2} \hat{\mathcal{L}}_{\hat{\mathcal{D}}_{\hat{H}} \hat{H}_{1}} \frac{\partial^{2} \hat{\mathscr{R}}_{\hat{H}}(z)}{\partial z^{2}}-\frac{1}{6}\left(\frac{i}{\hbar}\right)^{3} \hat{\mathcal{L}}_{\hat{\mathcal{D}}_{\hat{H}}^{2} \hat{H}_{1}} \frac{\partial^{3} \hat{\mathscr{R}}_{\hat{H}}(z)}{\partial z^{3}}+\ldots
\end{aligned}
$$

The derivative of superprojector $(\partial / \partial \alpha) \hat{\mathcal{P}}_{\hat{H}}$ can be obtained as the residue of this expression at $z=0$. In our case of an isolated point spectrum, the superresolvent is a meromorphic function, and therefore the residue of any of its derivatives with respect to $z$ vanishes identically. As a result, the superprojector $\hat{\mathcal{P}}_{\hat{H}}$ transforms canonically under perturbation:

$$
\frac{\partial}{\partial \alpha} \hat{\mathcal{P}}_{\hat{H}}=\hat{\mathcal{P}}_{\hat{H}} \hat{\mathcal{L}}_{\hat{\mathcal{S}}_{\hat{H}} \hat{H}_{1}}-\hat{\mathcal{L}}_{\hat{\mathcal{S}}_{\hat{H}} \hat{H}_{1}} \hat{\mathcal{P}}_{\hat{H}}
$$

This identity holds to all perturbation orders.

\section{AN EXPLICIT EXPRESSION FOR A GENERATOR}

\section{A. Ordered exponentials}

Ordered exponentials were introduced in quantum field theory by Dyson ${ }^{18}$ in 1949 . They parametrize an $\alpha$-dependent unitary transformation $\hat{U}(\alpha)$

$$
|\psi(0)\rangle=\hat{U}|\widetilde{\psi}(\alpha)\rangle, \quad \hat{\widetilde{H}}=\hat{U}^{-1} \hat{H} \hat{U}
$$


Kato expansion in quantum canonical perturbation theory

and its inverse $\hat{U}^{\dagger}=\hat{U}^{-1}$ using direct and inverse Hamiltonian flows in "time" $\alpha$ with some Hermitian generator $\hat{G}(\alpha)$ :

$$
\frac{\partial}{\partial \alpha} \hat{U}=i \hat{G}(\alpha) \hat{U}, \quad \frac{\partial}{\partial \alpha} \hat{U}^{\dagger}=-i \hat{U}^{\dagger} \hat{G}
$$

In classical mechanics the ordered exponentials are known as Lie-Deprit transforms ${ }^{19}$. Their applications to quantum canonical perturbation theory are discussed by $\mathrm{Ali}^{20}$ and $\mathrm{Scherer}^{21}$. We adopt the notation by Suzuki ${ }^{22}$ for the direct and inverse ordered exponentials:

$$
\hat{U}=\exp _{+}\left(i \int_{0}^{\alpha} \hat{G} d s\right), \quad \hat{U}^{\dagger}=\exp _{-}\left(-i \int_{0}^{\alpha} \hat{G} d s\right) .
$$

The integral signs here have only notational meaning as a reminiscence of the Dyson series.

We will mostly use the superoperatorial ordered exponentials that satisfy the equations:

$$
\frac{\partial}{\partial \alpha} \hat{\mathbf{U}}(\alpha)=i \hat{\mathcal{L}}_{\hat{G}(\alpha)} \hat{\mathbf{U}}, \quad \frac{\partial}{\partial \alpha} \hat{\mathbf{V}}(\alpha)=-i \hat{\mathbf{V}} \hat{\mathcal{L}}_{\hat{G}} .
$$

Analogous to the Hausdorff identity, they are factorizable as follows ${ }^{23}$ :

$$
\begin{aligned}
& \hat{\mathbf{U}}(\alpha) \hat{F}=\exp _{+}\left(i \int_{0}^{\alpha} \hat{\mathcal{L}}_{\hat{G}} d s\right) \hat{F}=\exp _{+}\left(i \int_{0}^{\alpha} \hat{G} d s\right) \hat{F} \exp _{-}\left(-i \int_{0}^{\alpha} \hat{G} d s\right), \\
& \hat{\mathbf{V}}(\alpha) \hat{F}=\exp _{-}\left(-i \int_{0}^{\alpha} \hat{\mathcal{L}}_{\hat{G}} d s\right) \hat{F}=\exp _{-}\left(-i \int_{0}^{\alpha} \hat{G} d s\right) \hat{F} \exp _{+}\left(i \int_{0}^{\alpha} \hat{G} d s\right) .
\end{aligned}
$$

Therefore, the transformed Hamiltonian can be written as $\hat{\widetilde{H}}=\exp _{-}\left(-i \int_{0}^{\alpha} \hat{\mathcal{L}}_{\hat{G}} d s\right) \hat{H}$.

In quantum physics the ordered exponentials are usually computed by the Dyson series ${ }^{18}$. But canonical perturbation theory uses their representation as a power series:

$$
\exp _{+}\left(i \int_{0}^{\alpha} \hat{\mathcal{L}}_{\hat{G}} d s\right)=\sum_{n=0}^{\infty} \alpha^{n} \hat{\mathbf{U}}_{n}, \quad \exp _{-}\left(-i \int_{0}^{\alpha} \hat{\mathcal{L}}_{\hat{G}} d s\right)=\sum_{n=0}^{\infty} \alpha^{n} \hat{\mathbf{V}}_{n} .
$$

Substituting these series and $\hat{G}(\alpha)=\sum_{n=0}^{\infty} \alpha^{n} \hat{G}_{n}$ into the equations (9), Deprit obtained the relations for the coefficients:

$$
\hat{\mathbf{U}}_{n}=\frac{i}{n} \sum_{k=0}^{n-1} \hat{\mathcal{L}}_{\hat{G}_{n-k-1}} \hat{\mathbf{U}}_{k}, \quad \hat{\mathbf{V}}_{n}=-\frac{i}{n} \sum_{k=0}^{n-1} \hat{\mathbf{V}}_{k} \hat{\mathcal{L}}_{\hat{G}_{n-k-1}},
$$

and developed the "triangular" computational algorithm ${ }^{19}$.

Iterations of (10) yield non-recursive formulas ${ }^{24}$ :

$$
\begin{aligned}
\hat{\mathbf{U}}_{n} & =\sum_{\substack{\left(m_{1}, \ldots, m_{r}\right) \\
n>m_{1}>m_{2}>\cdots>m_{r}}} i^{r+1} \frac{\hat{\mathcal{L}}_{\hat{G}_{n-m_{1}-1}}}{n} \frac{\hat{\mathcal{L}}_{\hat{G}_{m_{1}-m_{2}-1}}}{m_{1}} \cdots \frac{\hat{\mathcal{L}}_{\hat{G}_{m_{r}-1}}}{m_{r}}, \\
\hat{\mathbf{V}}_{n} & =\sum_{\substack{\left(m_{1}, \ldots, m_{r}\right) \\
n>m_{1}>m_{2}>\cdots>m_{r}}}(-i)^{r+1} \frac{\hat{\mathcal{L}}_{\hat{G}_{m_{r}-1}}}{m_{r}} \cdots \frac{\hat{\mathcal{L}}_{\hat{G}_{m_{1}-m_{2}-1}}}{m_{1}} \frac{\hat{\mathcal{L}}_{\hat{G}_{n-m_{1}-1}}}{n} .
\end{aligned}
$$


Kato expansion in quantum canonical perturbation theory

Here, the sum runs over all sets of integers $\left(m_{1}, \ldots, m_{r}\right)$, satisfying $n>m_{1}>\cdots>m_{r}>0$. In the first orders:

$$
\begin{aligned}
& \exp _{+}\left(i \int_{0}^{\alpha} \hat{\mathcal{L}}_{\hat{G}} d s\right)=1+i \alpha \hat{\mathcal{L}}_{\hat{G}_{0}}-\frac{\alpha^{2}}{2}\left(\hat{\mathcal{L}}_{\hat{G}_{0}}^{2}-i \hat{\mathcal{L}}_{\hat{G}_{1}}\right) \\
&-i \frac{\alpha^{3}}{6}\left(\hat{\mathcal{L}}_{\hat{G}_{0}}^{3}-i \hat{\mathcal{L}}_{\hat{G}_{0}} \hat{\mathcal{L}}_{\hat{G}_{1}}-2 i \hat{\mathcal{L}}_{\hat{G}_{1}} \hat{\mathcal{L}}_{\hat{G}_{0}}-2 \hat{\mathcal{L}}_{\hat{G}_{2}}\right)+\mathrm{O}\left(\alpha^{3}\right), \\
& \exp _{-}\left(-i \int_{0}^{\alpha} \hat{\mathcal{L}}_{\hat{G}} d s\right)=1-i \alpha \hat{\mathcal{L}}_{\hat{G}_{0}}-\frac{\alpha^{2}}{2}\left(\hat{\mathcal{L}}_{\hat{G}_{0}}^{2}+i \hat{\mathcal{L}}_{\hat{G}_{1}}\right) \\
&+i \frac{\alpha^{3}}{6}\left(\hat{\mathcal{L}}_{\hat{G}_{0}}^{3}+2 i \hat{\mathcal{L}}_{\hat{G}_{0}} \hat{\mathcal{L}}_{\hat{G}_{1}}+i \hat{\mathcal{L}}_{\hat{G}_{1}} \hat{\mathcal{L}}_{\hat{G}_{0}}-2 \hat{\mathcal{L}}_{\hat{G}_{2}}\right)+\mathrm{O}\left(\alpha^{3}\right) .
\end{aligned}
$$

\section{B. Intertwining transformation}

The canonical identity (8) means that the ordered exponential with the generator $i \hat{\mathcal{S}}_{\hat{H}} \hat{H}_{1}$ :

$$
\begin{aligned}
&|\psi(0)\rangle=\exp _{+}\left(i \int_{0}^{\alpha} i \hat{\mathcal{S}}_{\hat{H}} \hat{H}_{1} d s\right)|\widetilde{\psi}(\alpha)\rangle, \\
& \hat{\tilde{H}} \quad=\exp _{-}\left(-i \int_{0}^{\alpha} \hat{\mathcal{L}}_{i \hat{\mathcal{S}}_{\hat{H}} \hat{H}_{1}} d s\right) \hat{H},
\end{aligned}
$$

unitarily connects the perturbed and unperturbed superprojectors:

$$
\hat{\mathcal{P}}_{\hat{H}}=\exp _{+}\left(i \int_{0}^{\alpha} \hat{\mathcal{L}}_{i \hat{\boldsymbol{S}}_{\hat{H}} \hat{H}_{1}} d s\right) \hat{\mathcal{P}}_{\hat{H}_{0}} \exp _{-}\left(-i \int_{0}^{\alpha} \hat{\mathcal{L}}_{i \hat{\mathcal{S}}_{\hat{H}} \hat{H}_{1}} d s\right)
$$

Since this transformation intertwines the superprojectors

$$
\exp _{-}\left(-i \int_{0}^{\alpha} \hat{\mathcal{L}}_{i \hat{\mathcal{S}}_{\hat{H}} \hat{H}_{1}} d s\right) \hat{\mathcal{P}}_{\hat{H}}=\hat{\mathcal{P}}_{\hat{H}_{0}} \exp _{-}\left(-i \int_{0}^{\alpha} \hat{\mathcal{L}}_{i \hat{\mathcal{S}}_{\hat{H}} \hat{H}_{1}} d s\right)
$$

and $\hat{\mathcal{P}}_{\hat{H}} \hat{H}=\hat{H}$, the transformed Hamiltonian becomes block-diagonal:

$$
\hat{\widetilde{H}}=\exp _{-}\left(-i \int_{0}^{\alpha} \hat{\mathcal{L}}_{i \hat{\mathcal{S}}_{\hat{H}} \hat{H}_{1}} d s\right) \hat{\mathcal{P}}_{\hat{H}} \hat{H}=\hat{\mathcal{P}}_{\hat{H}_{0}} \exp _{-}\left(-i \int_{0}^{\alpha} \hat{\mathcal{L}}_{i \hat{\mathcal{S}}_{\hat{H}} \hat{H}_{1}} d s\right) \hat{H}=\hat{\mathcal{P}}_{\hat{H}_{0}} \hat{\widetilde{H}} .
$$

We have thus shown that the ordered exponential with the generator

$$
\hat{G}=i \hat{\mathcal{S}}_{\hat{H}} \hat{H}_{1}=i \sum_{n=0}^{\infty}(-1)^{n+1} \alpha^{n}(\sum_{\substack{\sum p_{j}=\mathbf{n}+1 \\ p_{j} \geq 0}} \hat{\mathcal{R}}_{\hat{H}_{0}}^{\left(p_{n+1}\right)} \underbrace{\hat{\mathcal{L}}_{\hat{H}_{1}} \hat{\boldsymbol{\mathcal { R }}}_{\hat{H}_{0}}^{\left(p_{n}\right)} \ldots \hat{\boldsymbol{\mathcal { R }}}_{\hat{H}_{0}}^{\left(p_{2}\right)} \hat{\mathcal{L}}_{\hat{H}_{1}}}_{n \text { times }} \hat{\boldsymbol{\mathcal { R }}}_{\hat{H}_{0}}^{\left(p_{1}\right)} \hat{H}_{1})
$$

formally block-diagonalizes the Hamiltonian in all orders in $\alpha$.

The above formula can be extended to systems with degenerate energy levels, or to unbounded perturbation in the form of an asymptotic series. It may be shown ${ }^{9}$ that an expression for $\hat{G}$ truncated at order $N$ results in a block-diagonal Hamiltonian up to $\mathrm{O}\left(\alpha^{N+1}\right)$. 
For clarity, we here use formal "analytic" expressions. But for degenerate systems, it must always be remembered that these expressions must be converted straightforwardly into truncated sums, and all equalities hold up to $\mathrm{O}\left(\alpha^{N+1}\right)$.

In the first perturbative orders, the generator

$$
\begin{aligned}
\hat{G}=i \hat{\mathcal{S}} \hat{H}_{1}-i \alpha\left(\hat{\mathcal{S}} \hat{\mathcal{L}}_{\hat{H}_{1}} \hat{\mathcal{S}}-\hat{\mathcal{S}}^{2} \hat{\mathcal{L}}_{\hat{H}_{1}} \hat{\mathcal{P}}-\hat{\mathcal{P}} \hat{\mathcal{L}}_{\hat{H}_{1}} \hat{\mathcal{S}}^{2}\right) \hat{H}_{1} \\
+i \alpha^{2}\left(\hat{\mathcal{S}} \hat{\mathcal{L}}_{\hat{H}_{1}} \hat{\mathcal{S}} \hat{\mathcal{L}}_{\hat{H}_{1}} \hat{\mathcal{S}}-\hat{\mathcal{S}} \hat{\mathcal{L}}_{\hat{H}_{1}} \hat{\mathcal{S}}^{2} \hat{\mathcal{L}}_{\hat{H}_{1}} \hat{\mathcal{P}}-\hat{\mathcal{S}} \hat{\mathcal{L}}_{\hat{H}_{1}} \hat{\mathcal{P}} \hat{\mathcal{L}}_{\hat{H}_{1}} \hat{\mathcal{S}}^{2}-\hat{\mathcal{S}}^{2} \hat{\mathcal{L}}_{\hat{H}_{1}} \hat{\mathcal{S}} \hat{\mathcal{L}}_{\hat{H}_{1}} \hat{\mathcal{P}}\right. \\
-\hat{\mathcal{S}}^{2} \hat{\mathcal{L}}_{\hat{H}_{1}} \hat{\mathcal{P}} \hat{\mathcal{L}}_{\hat{H}_{1}} \hat{\mathcal{S}}-\hat{\mathcal{P}} \hat{\mathcal{L}}_{\hat{H}_{1}} \hat{\mathcal{S}} \hat{\mathcal{L}}_{\hat{H}_{1}} \hat{\mathcal{S}}^{2}-\hat{\mathcal{P}} \hat{\mathcal{L}}_{\hat{H}_{1}} \hat{\mathcal{S}}^{2} \hat{\mathcal{L}}_{\hat{H}_{1}} \hat{\mathcal{S}}+\hat{\mathcal{S}}^{3} \hat{\mathcal{L}}_{\hat{H}_{1}} \hat{\mathcal{P}} \hat{\mathcal{L}}_{\hat{H}_{1}} \hat{\mathcal{P}} \\
+ \\
\left.+\hat{\mathcal{P}} \hat{\mathcal{L}}_{\hat{H}_{1}} \hat{\mathcal{S}}^{3} \hat{\mathcal{L}}_{\hat{H}_{1}} \hat{\mathcal{P}}+\hat{\mathcal{P}} \hat{\mathcal{L}}_{\hat{H}_{1}} \hat{\mathcal{P}} \hat{\mathcal{L}}_{\hat{H}_{1}} \hat{\mathcal{S}}^{3}\right) \hat{H}_{1}+\mathrm{O}\left(\alpha^{3}\right)
\end{aligned}
$$

yields the following block-diagonal effective Hamiltonian up to $\alpha^{4}$ :

$$
\begin{array}{r}
\hat{\widetilde{H}}=\hat{H}_{0}+\alpha \hat{\mathcal{P}} \hat{H}_{1}-\frac{\alpha^{2}}{2} \hat{\mathcal{P}} \hat{\mathcal{L}}_{\hat{H}_{1}} \hat{\mathcal{S}} \hat{H}_{1}+\alpha^{3}\left(\frac{1}{3} \hat{\mathcal{P}} \hat{\mathcal{L}}_{\hat{H}_{1}} \hat{\mathcal{\mathcal { S }}} \hat{\mathcal{L}}_{\hat{H}_{1}} \hat{\mathcal{\mathcal { S }}}-\frac{1}{6} \hat{\mathcal{\mathcal { P }}} \hat{\mathcal{L}}_{\hat{H}_{1}} \hat{\mathcal{S}}^{2} \hat{\mathcal{L}}_{\hat{H}_{1}} \hat{\mathcal{P}}\right) \hat{H}_{1} \\
+\alpha^{4}\left(\frac{1}{6} \hat{\mathcal{P}} \hat{\mathcal{L}}_{\hat{H}_{1}} \hat{\mathcal{S}} \hat{\mathcal{L}}_{\hat{H}_{1}} \hat{\mathcal{S}}^{2} \hat{\mathcal{L}}_{\hat{H}_{1}} \hat{\mathcal{P}}-\frac{1}{4} \hat{\mathcal{P}} \hat{\mathcal{L}}_{\hat{H}_{1}} \hat{\mathcal{S}} \hat{\mathcal{L}}_{\hat{H}_{1}} \hat{\mathcal{S}} \hat{\mathcal{L}}_{\hat{H}_{1}} \hat{\mathcal{S}}+\frac{1}{12} \hat{\mathcal{P}} \hat{\mathcal{L}}_{\hat{H}_{1}} \hat{\mathcal{S}}^{2} \hat{\mathcal{L}}_{\hat{H}_{1}} \hat{\mathcal{S}} \hat{\mathcal{L}}_{\hat{H}_{1}} \hat{\mathcal{P}}\right. \\
+\frac{1}{8} \hat{\mathcal{P}} \hat{\mathcal{L}}_{\hat{H}_{1}} \hat{\mathcal{S}}^{2} \hat{\mathcal{L}}_{\hat{H}_{1}} \hat{\mathcal{P}} \hat{\mathcal{L}}_{\hat{H}_{1}} \hat{\mathcal{S}}+\frac{1}{4} \hat{\mathcal{P}} \hat{\mathcal{L}}_{\hat{H}_{1}} \hat{\mathcal{P}} \hat{\mathcal{L}}_{\hat{H}_{1}} \hat{\mathcal{S}}^{2} \hat{\mathcal{L}}_{\hat{H}_{1}} \hat{\mathcal{S}}+\frac{1}{4} \hat{\mathcal{P}} \hat{\mathcal{L}}_{\hat{H}_{1}} \hat{\mathcal{P}} \hat{\mathcal{L}}_{\hat{H}_{1}} \hat{\mathcal{\mathcal { S }}} \hat{\mathcal{L}}_{\hat{H}_{1}} \hat{\mathcal{S}}^{2} \\
\left.-\frac{1}{6} \hat{\mathcal{P}} \hat{\mathcal{L}}_{\hat{H}_{1}} \hat{\mathcal{P}} \hat{\mathcal{L}}_{\hat{H}_{1}} \hat{\mathcal{S}}^{3} \hat{\mathcal{L}}_{\hat{H}_{1}} \hat{\mathcal{P}}-\frac{1}{4} \hat{\mathcal{P}} \hat{\mathcal{L}}_{\hat{H}_{1}} \hat{\mathcal{P}} \hat{\mathcal{L}}_{\hat{H}_{1}} \hat{\mathcal{P}} \hat{\mathcal{L}}_{\hat{H}_{1}} \hat{\mathcal{S}}^{3}\right) \hat{H}_{1}+\mathrm{O}\left(\alpha^{5}\right) .
\end{array}
$$

Here we omitted the indices $\hat{H}_{0}$ for unperturbed operators $\hat{\mathcal{P}}_{\hat{H}_{0}}$ and $\hat{\mathcal{S}}_{\hat{H}_{0}}$. These expressions are more regular than corresponding formulas ${ }^{6,7,25}$ for standard CPT methods.

\section{General form of the generator}

Knowing that $\hat{\mathcal{P}}_{\hat{H}_{0}}$ and $\hat{\mathcal{P}}_{\hat{H}}$ are unitary connected and that this transformation blockdiagonalizes the Hamiltonian, the procedure of canonical perturbation theory can be viewed as the construction of unitary transformation, which connects unperturbed and perturbed superprojectors.

Let us now determine the general form of the generator of such a transformation. The perturbed $\hat{\mathcal{P}}_{\hat{H}}$ should satisfy the differential equation

$$
\frac{\partial}{\partial \alpha} \hat{\mathcal{P}}_{\hat{H}}=i \hat{\mathcal{L}}_{\hat{G}} \hat{\mathcal{P}}_{\hat{H}}-i \hat{\mathcal{P}}_{\hat{H}} \hat{\mathcal{L}}_{\hat{G}} \text {. }
$$

Application of this expression to the Hamiltonian $\hat{H}$ and the identities

$$
\hat{\mathcal{P}}_{\hat{H}} \hat{H}=\hat{H}, \quad \frac{\partial}{\partial \alpha}\left(\hat{\mathcal{P}}_{\hat{H}} \hat{H}\right)=\left(\frac{\partial}{\partial \alpha} \hat{\mathcal{P}}_{\hat{H}}\right) \hat{H}+\hat{\mathcal{P}}_{\hat{H}} \frac{\partial}{\partial \alpha} \hat{H}, \quad \frac{\partial}{\partial \alpha} \hat{H}=\hat{H}_{1},
$$


Kato expansion in quantum canonical perturbation theory

yield

$$
\left(1-\hat{\mathcal{P}}_{\hat{H}}\right) \hat{\mathcal{L}}_{\hat{H}} \hat{G}=i\left(1-\hat{\mathcal{P}}_{\hat{H}}\right) \hat{H}_{1}
$$

To solve this equation, it is sufficient to apply the $\hat{\mathcal{S}}_{\hat{H}}$ superoperator. Therefore, the general form of the generator of intertwining unitary transformation is

$$
\hat{G}=i \hat{\mathcal{S}}_{\hat{H}} \hat{H}_{1}+\hat{\mathcal{P}}_{\hat{H}} \hat{F}
$$

where $\hat{F}(\alpha)$ may be any Hermitian operator. This is the main result of this work. This formula provides a non-recursive expression for the generator of the block-diagonalizing transformation and defines its ambiguity.

The choice of $\hat{\mathcal{P}}_{\hat{H}} \hat{F}$ is the uniqueness condition ${ }^{26}$. It is natural to choose $\hat{F} \equiv 0$ or $\hat{\mathcal{P}}_{\hat{H}} \hat{G}=0$. This is not equal to the "off-diagonal" condition $\hat{\mathcal{P}}_{\hat{H}_{0}} \hat{G}_{P}=0$ traditionally used in canonical perturbation theory ${ }^{5}$. Because $\hat{\mathcal{L}}_{\hat{\mathcal{P}}_{\hat{H}} \hat{F}} \hat{\mathcal{P}}_{\hat{H}}=\hat{\mathcal{P}}_{\hat{H}} \hat{\mathcal{L}}_{\hat{\mathcal{P}}_{\hat{H}} \hat{F}}$, the superprojector $\hat{\mathcal{P}}_{\hat{H}}$ is itself insensitive to this choice.

We can conclude that the generators of block-diagonalizing transformations can differ by a function belonging to a continuation of the algebra of integrals of unperturbed system.

Effective Hamiltonians that were block-diagonalized using different uniqueness conditions $\hat{F}_{1}$ and $\hat{F}_{2}$ are connected by unitary transformation:

$$
\hat{\mathbf{U}}_{21}=\exp _{-}\left(-i \int_{0}^{\alpha} \hat{\mathcal{L}}_{\hat{G}_{2}} d s\right) \exp _{+}\left(i \int_{0}^{\alpha} \hat{\mathcal{L}}_{\hat{G}_{1}} d s\right)
$$

Let us find its generator:

$$
\begin{aligned}
\frac{\partial}{\partial \alpha} \hat{\mathbf{U}}_{21} & =i \exp _{-}\left(-i \int_{0}^{\alpha} \hat{\mathcal{L}}_{\hat{G}_{2}} d s\right)\left(\hat{\mathcal{L}}_{\hat{G}_{1}}-\hat{\mathcal{L}}_{\hat{G}_{2}}\right) \exp \left(i \int_{0}^{\alpha} \hat{\mathcal{L}}_{\hat{G}_{1}} d s\right) \\
& =i \exp _{-}\left(-i \int_{0}^{\alpha} \hat{\mathcal{L}}_{\hat{G}_{2}} d s\right) \hat{\mathcal{L}}_{\hat{\mathcal{P}}_{\hat{H}}\left(\hat{F}_{1}-\hat{F}_{2}\right)} \exp _{+}\left(i \int_{0}^{\alpha} \hat{\mathcal{L}}_{\hat{G}_{1}} d s\right) \\
& =i \hat{\mathcal{L}}_{\text {exp }_{-}\left(-i \int_{0}^{\alpha} \hat{\mathcal{L}}_{\hat{G}_{2}} d s\right) \hat{\mathcal{P}}_{\hat{H}}\left(\hat{F}_{1}-\hat{F}_{2}\right)} \hat{\mathbf{U}}_{21} .
\end{aligned}
$$

Because of the intertwining relation (11), the generator of $\hat{\mathbf{U}}_{21}$ is always block-diagonal:

$$
\hat{G}_{21}=\exp _{-}\left(-i \int_{0}^{\alpha} \hat{\mathcal{L}}_{\hat{G}_{2}} d s\right) \hat{\mathcal{P}}_{\hat{H}}\left(\hat{F}_{1}-\hat{F}_{2}\right)=\hat{\mathcal{P}}_{\hat{H}_{0}} \exp _{-}\left(-i \int_{0}^{\alpha} \hat{\mathcal{L}}_{i \hat{\mathcal{S}}_{\hat{H}} \hat{H}_{1}+\hat{\mathcal{P}}_{\hat{H}} \hat{F}_{2}} d s\right)\left(\hat{F}_{1}-\hat{F}_{2}\right)
$$

This is the well-known result that the effective Hamiltonian is determined up to blockdiagonal transformation ${ }^{2}$. Such ambiguity does not affect the perturbed eigenvalues. 
Kato expansion in quantum canonical perturbation theory

\section{COMPUTATIONAL ASPECTS}

A major difference between this and standard canonical perturbation algorithms by Van Vleck $^{4}$, Primas ${ }^{5}$, etc., is the explicit non-recursive formulas. Traditionally, perturbation computations block-diagonalize the Hamiltonian order by order. In contrast, we compute the generator $\hat{G}=i \hat{\mathcal{S}}_{\hat{H}} \hat{H}_{1}$ up to the desired order directly. Then, the ordered exponential block-diagonalizes the Hamiltonian.

\section{A. An explicit algorithm for the generator}

Let us introduce the superoperators

$$
\hat{\mathcal{Z}}_{n}^{m}=(-1)^{n+1} \sum_{\substack{p_{1}+\ldots+p_{n+1}=m \\ p_{j} \geq 0}} \hat{\mathcal{R}}_{H_{0}}^{\left(p_{n+1}\right)} \underbrace{\hat{\mathcal{L}}_{\hat{H}_{1}} \hat{\mathcal{R}}_{H_{0}}^{\left(p_{n}\right)} \ldots \hat{\mathcal{R}}_{H_{0}}^{\left(p_{2}\right)} \hat{\mathcal{L}}_{\hat{H}_{1}}}_{n \text { times }} \hat{\mathcal{R}}_{H_{0}}^{\left(p_{1}\right)} .
$$

Here, the sum runs over all possible placements of $m$ superoperators $\hat{\mathcal{S}}_{H_{0}}$ in $n+1$ sets. The simplest such expressions are $\hat{\mathcal{Z}}_{0}^{0}=\hat{\mathcal{P}}_{H_{0}}$, and $\hat{\mathcal{Z}}_{0}^{m}=-\hat{\mathcal{S}}_{H_{0}}^{m}$ for $m>0$. We have already discussed their computation in section II.

The following recursive relation

$$
\hat{\mathcal{Z}}_{n}^{m}=\sum_{k=0}^{m} \hat{\mathcal{Z}}_{0}^{m-k} \hat{\mathcal{L}}_{\hat{H}_{1}} \hat{\mathcal{Z}}_{n-1}^{k}
$$

leads to an efficient algorithm for the generator $\hat{G}=i \hat{\mathcal{S}}_{H} \hat{H}_{1}$. We consider the square table:

$$
\begin{array}{cccccc}
\hat{F}_{0}^{0} & \hat{\boldsymbol{F}}_{0}^{1} & \hat{F}_{0}^{2} & \ldots & \hat{F}_{0}^{N} & \hat{F}_{0}^{N+1} \\
\hat{F}_{1}^{0} & \hat{F}_{1}^{1} & \hat{\boldsymbol{F}}_{1}^{2} & \ldots & \hat{F}_{1}^{N} & \hat{F}_{1}^{N+1} \\
& \ldots & & & & \\
\hat{F}_{N-1}^{0} & \hat{F}_{N-1}^{1} & \hat{F}_{N-1}^{2} & \ldots & \boldsymbol{F}_{N-1}^{\hat{N}} & \hat{F}_{N-1}^{N+1} \\
& & & & & \boldsymbol{F}_{N}^{\hat{N}+1}
\end{array}
$$

Here the first row is

$$
\hat{F}_{0}^{0}=\hat{\mathcal{Z}}_{0}^{0} \hat{H}_{1}=\hat{\mathcal{P}}_{H_{0}} \hat{H}_{1}, \quad \hat{F}_{0}^{m}(\mathbf{x})=\hat{\mathcal{Z}}_{0}^{m} \hat{H}_{1}=-\hat{\mathcal{S}}_{H_{0}}^{m} \hat{H}_{1},
$$

and each subsequent row is generated from the previous row according to the rule

$$
\hat{F}_{n+1}^{m}=\sum_{k=0}^{m} \hat{\mathcal{Z}}_{0}^{m-k} \hat{\mathcal{L}}_{\hat{H}_{1}} \hat{F}_{n}^{k}
$$

The generator truncated at order $\alpha^{N+1}$ is given by $\hat{G}_{[N]}=i \sum_{n=0}^{N} \alpha^{n} \hat{F}_{n}^{n+1}$. 
Kato expansion in quantum canonical perturbation theory

\section{B. Computation of the ordered exponential}

In classical mechanics the ordered exponentials are usually computed using the Deprit "triangle" 19 . However, we have observed that for all of our quantum examples the following algorithm is faster.

Up to $\mathrm{O}\left(\alpha^{N+1}\right)$ the transform of general perturbed Hamiltonian $\hat{H}=\sum_{n=0}^{N} \alpha^{n} \hat{H}_{n}$ can be written in the following form:

$$
\hat{\widetilde{H}}=\exp _{-}\left(-i \int_{0}^{\alpha} \hat{\mathcal{L}}_{\hat{G}} d s\right) \hat{H}=\left(\sum_{n=0}^{N} \alpha^{n} \hat{\mathbf{V}}_{n}\right) \hat{H}=\sum_{n=0}^{N} \hat{\mathbf{V}}_{n} \hat{\widetilde{F}}_{n}^{(N)} .
$$

Here we introduced ancillary double-indexed operators $\hat{\widetilde{F}}_{n}^{(N)}=\sum_{k=n}^{N} \alpha^{k} \hat{H}_{k-n}$.

The Deprit relation (10) allows us to express repeatedly the superoperators $\hat{\mathbf{V}}_{n}$ by means of $\hat{\mathbf{V}}_{\text {less then } n}$ :

$$
\hat{\widetilde{H}}=\hat{\mathbf{V}}_{N} \hat{\widetilde{F}}_{N}^{(N)}+\sum_{k=0}^{N-1} \hat{\mathbf{V}}_{k} \hat{\widetilde{F}}_{k}^{(N)}=\sum_{k=0}^{N-1} \hat{\mathbf{V}}_{k} \hat{\widetilde{F}}_{k}^{(N-1)}=\ldots=\sum_{k=0}^{n} \hat{\mathbf{V}}_{k} \hat{\widetilde{F}}_{k}^{(n)}=\ldots
$$

where the operators $\hat{\widetilde{F}}_{k}^{(n)}, n=N-1, \ldots, 0$ are computed using the following relation:

$$
\hat{\widetilde{F}}_{k}^{(n)}=\hat{\widetilde{F}}_{k}^{(n+1)}-\frac{i}{n+1} \hat{\mathcal{L}}_{\hat{G}_{n-k}} \hat{\widetilde{F}}_{n+1}^{(n+1)}, \quad k=0, \ldots, n
$$

Finally, all the superoperators $\hat{\mathbf{V}}$ disappear and we obtain the transformed Hamiltonian:

$$
\hat{\widetilde{H}}=\hat{\widetilde{F}}_{0}^{(0)} .
$$

\section{Examples and comparison with other methods}

The explicit expression is important from a general mathematical standpoint because it systematizes and simplifies the perturbation expansion. Moreover, it provides a sufficiently effective computational algorithm. Here we compare the computer times of block-diagonalization using the explicit expression (12) with those of the Van Vleck ${ }^{4}$ and Magnus $^{27,28}$ approaches.

The Van Vleck method block-diagonalizes the Hamiltonian using a chain of exponents:

$$
\hat{\widetilde{H}}=e^{-i \alpha^{n} \hat{G}_{n-1}} \ldots e^{-i \alpha \hat{G}_{0}} \hat{H} e^{i \alpha \hat{G}_{0}} \ldots e^{i \alpha^{n} \hat{G}_{n-1}}=e^{-i \alpha^{n} \hat{\mathcal{L}}_{\hat{G}_{n-1}}} \ldots e^{-i \alpha \hat{\mathcal{L}}_{\hat{G}_{0}} \hat{H}}
$$

In classical mechanics it is known as the Dragt-Finn transformation ${ }^{29}$. 
Kato expansion in quantum canonical perturbation theory

The Magnus method uses the superoperator exponent with an $\alpha$-dependent generator ${ }^{5}$ :

$$
\hat{\widetilde{H}}=e^{-i \sum_{1}^{n} \alpha^{k} \hat{G}_{k-1}} \hat{H} e^{i \sum_{1}^{n} \alpha^{k} \hat{G}_{k-1}}=e^{-i \sum_{1}^{n} \alpha^{k} \hat{\mathcal{L}}_{\hat{G}_{k-1}}} \hat{H}
$$

In classical mechanics this is known as the Hori algorithm ${ }^{30}$.

We have evaluated the efficiency of these methods for the following model systems:

- The one-dimensional oscillator with the quartic anharmonicity:

$$
\hat{H}=\frac{1}{2}\left(\hat{p}^{2}+\hat{q}^{2}\right)+\frac{\alpha}{4} \hat{q}^{4}
$$

The first orders of the block-diagonalized Hamiltonian are:

$\hat{\tilde{H}}=\hbar\left(\hat{N}+\frac{1}{2}\right)+\alpha \hbar^{2}\left(\frac{3}{8}\left(\hat{N}+\frac{1}{2}\right)^{2}+\frac{3}{32}\right)-\alpha^{2} \hbar^{3}\left(\frac{17}{64}\left(\hat{N}+\frac{1}{2}\right)^{3}+\frac{67}{256}\left(\hat{N}+\frac{1}{2}\right)\right)+\mathrm{O}\left(\alpha^{3}\right)$.

Here $\hat{N}=\hat{a}^{\dagger} \hat{a}$. This is the typical structure of the perturbation series for nondegenerate systems. It is worth noting that the effective diagonal Hamiltonians of such systems are identical for all the CPT methods.

- The Hénon-Heiles system. This is a two-dimensional system with the Hamiltonian

$$
\hat{H}=\frac{1}{2}\left(\hat{p}_{1}^{2}+\hat{q}_{1}^{2}+\hat{p}_{2}^{2}+\hat{q}_{2}^{2}\right)+\alpha\left(\hat{q}_{1}^{2} \hat{q}_{2}-\frac{1}{3} \hat{q}_{2}^{3}\right) .
$$

The block-diagonalized effective Hamiltonian is:

$$
\begin{aligned}
\hat{\tilde{H}}= & \left(\hbar-\frac{1}{9} \alpha^{2} \hbar^{2}-\frac{11}{108} \alpha^{4} \hbar^{3}\right)+\left(\hbar-\frac{2}{3} \alpha^{2} \hbar^{2}-\frac{61}{54} \alpha^{4} \hbar^{3}\right)\left(\hat{a}_{1}^{\dagger} \hat{a}_{1}+\hat{a}_{2}^{\dagger} \hat{a}_{2}\right) \\
& -\left(\frac{5}{12} \alpha^{2} \hbar^{2}+\frac{47}{48} \alpha^{4} \hbar^{3}\right)\left(\hat{a}_{1}^{\dagger 2} \hat{a}_{1}^{2}+\hat{a}_{2}^{\dagger 2} \hat{a}_{2}^{2}\right)-\left(\frac{7}{12} \alpha^{2} \hbar^{2}-\frac{7}{48} \alpha^{4} \hbar^{3}\right)\left(\hat{a}_{2}^{\dagger 2} \hat{a}_{1}^{2}+\hat{a}_{1}^{\dagger 2} \hat{a}_{2}^{2}\right) \\
& +\left(\frac{1}{3} \alpha^{2} \hbar^{2}-\frac{9}{4} \alpha^{4} \hbar^{3}\right) \hat{a}_{1}^{\dagger} \hat{a}_{1} \hat{a}_{2}^{\dagger} \hat{a}_{2} \\
& +\alpha^{4} \hbar^{3}\left(\frac{101}{432} \hat{a}_{1}^{\dagger 3} \hat{a}_{1}^{3}-\frac{161}{144} \hat{a}_{1}^{\dagger 3} \hat{a}_{1} \hat{a}_{2}^{2}-\frac{65}{16} \hat{a}_{1}^{\dagger 2} \hat{a}_{2}^{\dagger} \hat{a}_{1}^{2} \hat{a}_{2}+\frac{175}{144} \hat{a}_{1}^{\dagger 2} \hat{a}_{2}^{\dagger} \hat{a}_{2}^{3}\right. \\
& \left.-\frac{235}{432} \hat{a}_{2}^{\dagger 3} \hat{a}_{2}^{3}-\frac{161}{144} \hat{a}_{1}^{\dagger} \hat{a}_{2}^{\dagger 2} \hat{a}_{1}^{3}+\frac{47}{16} \hat{a}_{1}^{\dagger} \hat{a}_{2}^{\dagger 2} \hat{a}_{1} \hat{a}_{2}^{2}+\frac{175}{144} \hat{a}_{2}^{\dagger 3} \hat{a}_{1}^{2} \hat{a}_{2}\right)+\mathrm{O}\left(\alpha^{6}\right) .
\end{aligned}
$$

This expression could be further diagonalized using finite canonical transformations ${ }^{31}$.

All three CPT methods build near-identity unitary block-diagonalizing transformations. Such transformations are equivalent to ordered exponentials with different conditions $\hat{\mathcal{P}}_{\hat{H}} \hat{F}$. As a result, all the three effective block-diagonal Hamiltonians of the Hénon-Heiles system differ from each other starting from the $8^{\text {th }}$ perturbation order, and are connected by block-diagonal unitary transformations. 
Kato expansion in quantum canonical perturbation theory

The comparison of efficiency of these methods is not unambiguous. High order computations process large multi-gigabyte expressions containing millions of terms. The performance of operations with such expressions depends on the computer algebra system used, its internal optimizations, the server CPU, RAM, disk subsystem, etc. Even the relative efficiencies of methods may vary. Therefore, the following our results are only illustrative.

Figure 1 compares the computational times of block-diagonalization for the Hénon-Heiles system on an Oracle ${ }^{\mathrm{TM}}$ Exadata X2-2 server with Intel Xeon X5675 (3.06 GHz) processor using Form 4.1 computer algebra system ${ }^{12}$.

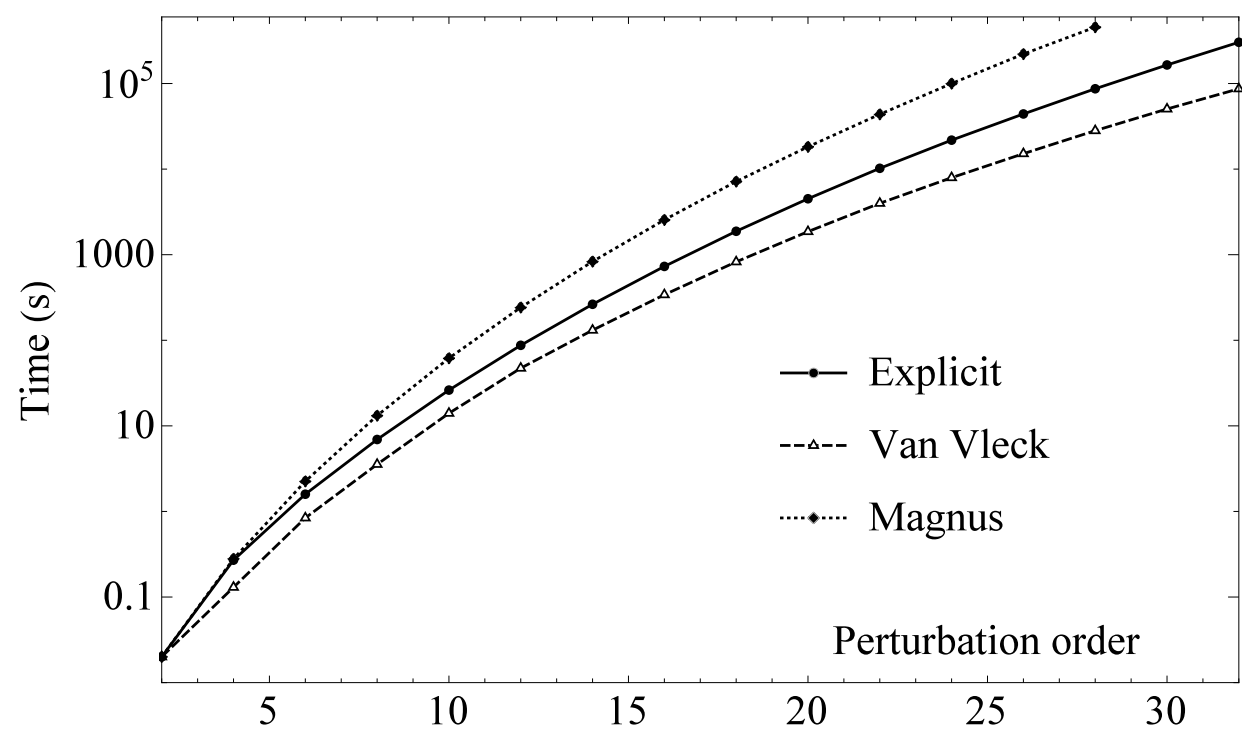

FIG. 1. Block-diagonalization time for Hénon-Heiles Hamiltonian.

The computation times for the quartic anharmonic oscillator follow the same pattern. In these particular cases the explicit algorithm is faster for high-orders computations than the Magnus expansion, but less efficient then the Van Vleck method.

\section{SUMMARY}

We have presented here an application of Kato resolvent expansion to quantum superoperatorial canonical perturbation theory. The canonical identity for the superresolvent allowed us to demonstrate unitary intertwining of perturbed and unperturbed averaging superprojectors. This leads to the explicit expression for the generator of the block-diagonalizing transformation in any perturbation order and systematic description of ambiguities in the generator and block-diagonalized Hamiltonian. 
Kato expansion in quantum canonical perturbation theory

We have also compared the computational efficiency of the explicit expression for the quartic anharmonic oscillator and the Hénon-Heiles system with the efficiencies of the Van Vleck and Magnus methods up to the $32^{\text {nd }}$ perturbation order.

\section{ACKNOWLEDGMENTS}

The author gratefully acknowledges Professor S. V. Klimenko, RDTEX Technical Support Centre Director S. P. Misiura, and V. V. Romanova for encouragement and support.

\section{REFERENCES}

${ }^{1}$ M. Born, W. Heisenberg, and P. Jordan, Z. Phys. 35, 557 (1926).

${ }^{2}$ C. E. Soliverez, Phys. Rev. A 24, 4 (1981).

${ }^{3}$ F. Jørgensen, Mol. Phys. 29, 1137 (1975).

${ }^{4}$ J. H. Van Vleck, Phys. Rev. 33, 467 (1929).

${ }^{5}$ H. Primas, Rev. Mod. Phys. 35, 710 (1963).

${ }^{6}$ D. J. Klein, J. Chem. Phys. 61, 786 (1974).

${ }^{7}$ V. G. Tyuterev and V. I. Perevalov, Chem. Phys. Lett. 74, 494 (1980).

${ }^{8}$ V. Tyuterev, S. Tashkun, M. Rey, R. Kochanov, A. Nikitin, and T. Delahaye, J. Phys. Chem. A 117, 13779 (2013).

${ }^{9}$ A. S. Nikolaev, Theor. Math. Phys. 182, 407 (2015).

${ }^{10}$ T. Kato, Perturbation Theory for Linear Operators (Springer-Verlag, New York, 1995).

${ }^{11}$ A. S. Nikolaev, "Supplementary demonstrations http://andreysnikolaev.wordpress.com/demo," . ${ }^{12}$ J. Kuipers, T. Ueda, J. A. M. Vermaseren, and J. Vollinga, Comput. Phys. Commun. 184, 1453 (2013).

${ }^{13}$ P.-O. Löwdin, Int. J. Quantum Chem. 22, 485 (1982).

${ }^{14}$ W. H. Shaffer, H. H. Nielsen, and L. H. Thomas, Phys. Rev. 56, 895 (1939).

${ }^{15}$ G. Birkhoff, Dynamical Systems (Amer. Math. Soc., New York, 1966).

${ }^{16}$ F. G. Gustavson, Astron. J. 71, 670 (1966).

${ }^{17}$ W. Liu and Z. Huang, Int. J. Theor. Phys. 52, 2578 (2013).

${ }^{18}$ F. J. Dyson, Phys. Rev. 75, 486 (1949).

${ }^{19}$ A. Deprit, Celestial Mech. 1, 12 (1969). 
Kato expansion in quantum canonical perturbation theory

${ }^{20}$ M. K. Ali, J. Math. Phys. 26, 2565 (1985).

${ }^{21}$ W. Scherer, J. Phys. A: Math. Gen. 27, 8231 (1994).

${ }^{22}$ M. Suzuki, J. Math. Phys. 26, 601 (1985).

${ }^{23}$ H. Araki, Ann. Sci. Éc. Norm. Supér. 6, 67 (1973).

${ }^{24}$ J. R. Cary, Phys. Rep. 79, 129 (1981).

${ }^{25}$ I. Shavitt and L. T. Redmon, J. Chem. Phys. 73, 5711 (1980).

${ }^{26}$ C. E. Soliverez, Phys. Lett. A 79, 410 (1980).

${ }^{27}$ W. Magnus, Commun. Pur. Appl. Math. 7, 649 (1954).

${ }^{28}$ S. Blanes, F. Casas, J. Oteo, and J. Ros, Phys. Rep. 470, 151 (2009).

${ }^{29}$ A. J. Dragt and J. M. Finn, J. Math. Phys. 17, 2215 (1976).

${ }^{30}$ G. Hori, Publ. Astron. Soc. Japan 18, 287 (1966).

${ }^{31}$ A. S. Nikolaev, J. Math. Phys. 37, 2643 (1996). 